\title{
Event-Based Model Predictive Control for the Cooperation of Distributed Agents
}

\author{
Alina Eqtami, Dimos V. Dimarogonas and Kostas J. Kyriakopoulos
}

\begin{abstract}
This paper proposes an event-based framework for the control of a team of cooperating distributed agents. The agents are dynamically decoupled and they are controlled locally by Nonlinear Model Predictive Controllers (NMPC). The event-driven framework allows for triggering the solution of the optimal control problem of the NMPC only when it is needed. The scheduling of the control updates for each of the agents depends on an error of the state information received from the neighboring agents. Sufficient conditions for triggering are provided and the results are illustrated through a simulated example.
\end{abstract}

\section{INTRODUCTION}

The control of many interacting subsystems has gained much interest in the recent years. Formulating the problem of control of such large-scale systems under a NMPC framework is an efficient approach because of the inherent virtues of these kind of controllers. NMPC can handle nonlinearities and offer the possibility of incorporating control and state constraints. Related results on NMPC for large-scale systems can be found in [5], [11], [13], [14] and in the review paper [15] and the number of papers quoted therein.

When implementing decentralized control laws, the communication schemes between interacting subsystems as well as the controllers' design, are aspects that should be taken into consideration. In the event-based approaches the decision for the execution of the control task depends on the state of the system. This methodology may lead to an overall reduction on the number of the control updates which might be desirable when the system has limited resources. Related works on event-based control can be found in [1], [2], [3], [8], [10], [17], [19], [20], and [21].

The computation of the control law of the NMPC controller is rather demanding particularly when large-scale systems are of consideration. Motivated by this fact, an event-based framework for this kind of controllers is investigated in order to reduce the number of times the control input should be computed. Under the proposed scheme the control law of the NMPC is not updated at each sampling instant but rather, the already computed control sequence is implemented to the plant until an event occurs. The problem addressed here is the control of a team of cooperating agents

Alina Eqtami and Kostas J. Kyriakopoulos are with the Control Systems Lab, Department of Mechanical Engineering, National Technica University of Athens, 9 Heroon Polytechniou Street, Zografou 15780 , Greece $\{a l i n a, k k y r i a @ m a i l . n t u a . g r\}$. Dimos V. Dimarogonas is with the ACCESS Linnaeus Centre, School of Electrical Engineering, KTH Royal Institute of Technology, Stockholm, Sweden. He is also affiliated with the KTH Centre for Autonomous Systems and is supported by the Swedish Research Council (VR) through contract 20093948. \{dimos@ee.kth.se\}. operating in the same environment. Each agent is a nonlinear discrete-time system and no dynamic coupling between the agents is assumed. The agents are controlled by local NMPC controllers which depend not only on local information, but also on the information of the neighboring agents.

The contribution of this paper relies in finding sufficient conditions for triggering in the case of a team of cooperative nonlinear subsystems. Each one of the subsystems has its own triggering condition which depends on the local state information and an error of the state information of their neighbors. The stability, and particularly the Input-to-State (ISS) stability, of a system of cooperating agents under NMPC has been presented in [5]. The authors consider the classic time-driven NMPC where the optimization problem is solved at each sampling instant. In this work we appropriately modify the formulation presented in [5] in order to reach a triggering condition. Moreover, unlike [5] where the predicted dynamics of the neighbors are considered to be of decreasing "importance" during the prediction horizon, in this work the error of the prediction is included in the triggering condition.

Even though the field of event-based NMPC is rather new, some relevant works have been presented. In [18] the NMPC framework for centralized continuous-time systems is event-driven, albeit a criterion for triggering was not provided. More recently, in [4], a triggering condition for a decentralized NMPC was given. That approach consisted of describing the effect of the interconnection among the subsystems as disturbances acting on local models and a robust NMPC approach was utilized in order to reach to the triggering condition. The aforementioned scheme is applicable to systems where the interaction between the agents is limited, opposed to our proposed framework, where the interactions between the agents are taken explicitly into account. Other related papers can be found in [7], [9], [16].

The remainder of the paper is organized as follows. In Section II, the problem statement for the cooperative control of distributed agents under local NMPC control laws is presented. The ISS stability properties and the sufficient conditions for triggering of each of the cooperating agents, along with a brief discussion on event-triggering in the presence of communication delays, are provided in Section III. In Section IV some simulation results are presented and Section V summarizes the results of this paper and indicates further research endeavors. 


\section{Problem Statement}

In the following, triggering conditions for distributed agents which operate in a common environment under local NMPC control laws, are going to be presented. Consider a general system which is composed by $M$ local subsystems. The dynamics of the subsystems are described by a nonlinear discrete-time equation

$$
x_{k+1}^{i}=f^{i}\left(x_{k}^{i}, u_{k}^{i}\right)
$$

with $k \in \mathbb{Z}_{>0}$ and $i=1, \ldots, M$. The state of subsystem $i$ is denoted by $x_{k}^{i} \in \mathbb{R}^{n^{i}}$, while $u_{k}^{i} \in \mathbb{R}^{m^{i}}$ denotes the control variable. Assume that $f^{i}(0,0)=0$ and suppose that the agents evolve on the same discrete-time space. The state and the control vectors are required to fulfill the following constraints

$$
x_{k}^{i} \in \mathscr{X}^{i} \quad u_{k}^{i} \in \mathscr{U}^{i}
$$

where $\mathscr{X}^{i}$ is a compact set of $\mathbb{R}^{n^{i}}$ and $\mathscr{U}^{i}$ is a compact set of $\mathbb{R}^{m^{i}}$, all of them containing the origin as an interior point.

Given the system (1), the predicted state is denoted by

$$
\hat{x}^{i}(k+l+1 \mid k)=f^{i}\left(\hat{x}^{i}(k+l \mid k), u_{k+l}^{i}\right)
$$

This notation will be equipped hereafter and it accounts for the predicted state at time $k+l+1$ with $l \in \mathbb{Z}_{\geq 0}$, based on the measurement of the state at time $k+l$ while using a control input $u_{k+l}^{i}$.

\section{A. NMPC for cooperative control}

As already mentioned, a distributed control structure is assumed in our scenario, where each one of the subsystems is controlled by a local NMPC controller. A partially connected framework is considered in this paper, i.e., the information is transmitted from any local controller, only to a given subset of the others. More precisely, each agent $\mathscr{A}^{i}, \forall i=1, \ldots, M$ exchanges state information with a set of neighboring agents $\mathscr{G}^{i} \triangleq\left\{\mathscr{A}^{j}, j \in G^{i}\right\}$, where $G^{i}$ denotes the set of indexes identifying the agents belonging to the set $\mathscr{G}^{i}$. The state information received by an agent $\mathscr{A}^{i}$ at time step $k$, can be written in stack vector form as

$$
w_{k}^{i} \triangleq \operatorname{col}\left(x_{k}^{j}, j \in G^{i}\right)
$$

with

$$
w_{k}^{i} \in \mathscr{W}^{i} \triangleq \Pi_{j \in G^{i}} \mathscr{X}^{j}
$$

Note that any agent knows the state of the agents in its neighborhood without delay. In a subsequent section, the presence of transmission delays is going to be discussed as well.

In the centralized NMPC the control law is computed by solving a finite-horizon, open-loop optimal control problem (OCP), based on the state measurement provided by the plant. In the distributed case though, each agent $\mathscr{A}^{i}$ solves an OCP based not only on its state measurements $x_{k}^{i}$, but also on the information vector of the neighbors $w_{k}^{i}$. The optimal problem, consists in minimizing, with respect to a control sequence $u_{F}^{i}(k) \triangleq\left[u^{i}(k \mid k), u^{i}(k+1 \mid k), \ldots, u^{i}\left(k+N^{i}-1 \mid k\right)\right]$, a cost function $J_{N}^{i}\left(x_{k}^{i}, u_{F}^{i}(k), w_{k}^{i}\right)$. Thus, the OCP for the system (1), is given by

$$
\begin{aligned}
& \min _{u_{F}^{i}(k)} J_{N}^{i}\left(x_{k}^{i}, u_{F}^{i}(k), w_{k}^{i}\right)= \\
& \min _{u_{F}^{i}(k)} \sum_{l=0}^{l=N^{i}-1}\left\{L^{i}\left(\tilde{x}^{i}(k+l \mid k), u^{i}(k+l \mid k)\right)\right. \\
& \left.+Q^{i}\left(\tilde{x}^{i}(k+l \mid k), w^{i}(k+l \mid k)\right)\right\}+V^{i}\left(\tilde{x}^{i}\left(k+N^{i} \mid k\right)\right)
\end{aligned}
$$

s.t.

$$
\begin{array}{ll}
\tilde{x}^{i}(k+l \mid k) \in \mathscr{X}^{i} & \forall l=1, \ldots, N^{i}-1 \\
u^{i}(k+l \mid k) \in \mathscr{U}^{i} & \forall l=0, \ldots, N^{i}-1 \\
w^{i}(k+l \mid k) \in \mathscr{W}^{i} & \forall l=0, \ldots, N^{i}-1 \\
\tilde{x}^{i}\left(k+N^{i} \mid k\right) \in \mathscr{X}_{f}^{i} &
\end{array}
$$

where $\mathscr{X}_{f}^{i}$ denotes the terminal constraint set and $\sim$ denotes the controller internal variables with $\tilde{x}^{i}(k \mid k)=x_{k}^{i}$. The positive integer $N^{i} \in \mathbb{Z}_{\geq 0}$, denotes the prediction horizon.

The vector $w^{i}(k+l \mid k)$ for $l=0, \ldots, N^{i}-1$, denotes the prediction of the neighbors' states. Since, only $w^{i}(k \mid k) \triangleq w_{k}^{i}$ is known to the agent $\mathscr{A}^{i}$, the following is assumed

$$
w^{i}(k+l \mid k)=w^{i}(k \mid k) \quad \forall l=0, \ldots, N^{i}-1
$$

Namely, each agent assumes that its neighbors maintain the same state during the prediction horizon. This assumption is utilized in order to solve the OCP, (6a)-(6e). Obviously, this is not the case due to the individual agent dynamics. The use of the event-triggered framework will enable us to overcome this limitation.

Some standard stability conditions for the design parameters of the NMPC must be introduced, in order to assert that NMPC strategy results in a stabilizing controller.

Assumption 1: The stage cost $L^{i}\left(x^{i}, u^{i}\right)$ is Lipschitz continuous in $\mathscr{X}^{i} \times \mathscr{U}^{i}$ and it holds that $L^{i}(0,0)=0$. Moreover, there is a $\mathscr{K}_{\infty}$-function $r^{i}$, such that $L^{i}\left(x_{k}^{i}, u_{k}^{i}\right) \geq r^{i}\left(\left\|x_{k}^{i}\right\|\right)$. Note, that a continuous function $r^{i}$ is said to belong to class $\mathscr{K}_{\infty}$ if $r^{i}=\infty$ and $r^{i}(x) \rightarrow \infty$ as $x \rightarrow \infty$.

Assumption 2: The running cost $Q^{i}\left(x^{i}, w^{i}\right)$ is such that $Q^{i}\left(x^{i}, w^{i}\right) \geq 0$. Moreover, $Q^{i}$ is Lipschitz continuous in $\mathscr{X}^{i} \times$ $\mathscr{W}^{i}$, with Lipschitz constants $L_{q x}^{i}$ and $L_{q w}^{i}$, respectively.

Assumption 3: Let the terminal set $\mathscr{X}_{f}^{i}$ be such that $\mathscr{X}_{f}^{i} \subset$ $\mathscr{X}^{i}, \mathscr{X}_{f}^{i}$ to be closed, and $0 \in \mathscr{X}_{f}^{i}$. Assume that there is a locally stabilizing controller $h^{i}\left(x_{k}^{i}\right)$ for the terminal set. Note, that $h^{i}\left(x_{k}^{i}\right) \in \mathscr{U}^{i}$, for all $x_{k}^{i} \in \mathscr{X}_{f}^{i}$. The associated Lyapunov function $V^{i}(\cdot)$ has the following property

$$
\begin{aligned}
& V^{i}\left(f^{i}\left(x_{k}^{i}, h^{i}\left(x_{k}^{i}\right)\right)\right)-V^{i}\left(x_{k}^{i}\right) \leq-L^{i}\left(x_{k}^{i}, h^{i}\left(x_{k}^{i}\right)\right) \\
& -Q^{i}\left(x_{k}^{i}, w_{k}^{i}\right) \forall x^{i} \in \mathscr{X}_{f}^{i} \text { and } \forall w^{i} \in \mathscr{W}^{i}
\end{aligned}
$$

\section{EVENT-BASED NMPC}

In this section a triggering condition for each one of the agents $\mathscr{A}^{i}$, will be provided. Before tackling this problem though, some concepts about the event-based approach for a distributed NMPC scheme will be given.

Consider a generic time-instant $k$. The solution of the OCP (6a)-(6e) provides an optimal control sequence $u^{i *}(k+l \mid k)$ 
for $l=0, \ldots, N^{i}-1$. In the classic NMPC strategy only the first term of the optimal solution $u^{i}(k \mid k) \triangleq u_{k}^{i}$ is applied to the system and the control law is updated for the next time-step $k+1$. In the event-based framework some portion of the optimal solution $\left[u^{i *}(k \mid k), \ldots, u^{i *}(k+\hat{l} \mid k)\right]$ with $\hat{l} \in$ $\left[0, N^{i}-1\right]$, is applied to the plant in an open-loop fashion, provided some stability conditions are fulfilled. The eventbased strategy is used in order to enlarge, as much as possible, the inter-calculation period of the NMPC. Note that the inter-calculation time is an integer multiple of the base sampling period. This results to the overall reduction of the control updates which is desirable in numerous occasions, for example energy consumption reasons.

\section{A. ISS Stability With Respect to Measurement Errors of the Neighbors}

In order to find a triggering condition for the distributed system under the NMPC control laws, the ISS properties of the systems will be used. A modification of the analysis proposed by [5] will be used in the following approach, in order to reach to the triggering condition of each of the agents.

Consider an event, triggered at time-step $k-1$, which provides an optimal sequence $u_{F}^{i *}(k-1)$. Consider also, control trajectories $\bar{u}_{F}^{i}(k+m)$, for time steps $m=0, \ldots, N^{i}-1$, based on the optimal solution in $k-1$,

$$
\begin{aligned}
& \bar{u}^{i}(k+t \mid k+m)= \\
& =\left\{\begin{aligned}
u^{i *}(k+t \mid k-1) & \text { for } t=m, \ldots, N^{i}-2 \\
h^{i}\left(\hat{x}^{i}\left(k+N^{i}-1 \mid k+m\right)\right) & \text { for } t=m+N^{i}-1
\end{aligned}\right.
\end{aligned}
$$

These control sequences are admissible and in general suboptimal. From the feasibility of $u_{F}^{i *}(k-1)$ it follows that for all $m=0, \ldots, N^{i}-1$ we have $\bar{u}^{i}(k+t \mid k+m) \in \mathscr{U}^{i}$ and $\hat{x}^{i}\left(k+N^{i} \mid k+m\right) \in \mathscr{X}_{f}^{i}$.

The optimal cost at the triggering instant $k-1$ is denoted by $J_{N}^{i *}(k-1)$ and the cost of the feasible sequence at a time step $t \in\left[0, N^{i}-1\right]$ is indicated by $\vec{J}_{N}^{i}(k+t)$. The difference between these costs is

$$
\Delta J_{t}^{i}=\vec{J}_{N}^{i}(k+t)-J_{N}^{i *}(k-1)
$$

The next lemma can now be stated

Lemma 1: Consider the system (1) subject to (2) and assume that Assumptions 1, 2, 3, hold. The difference (9) is bounded by

$$
\begin{aligned}
\Delta J_{t}^{i} & \leq\left(N^{i}-t-2\right) L_{q w}^{i} e_{w}^{i}(k+t \mid k-1) \\
& -\sum_{\rho=0}^{t}\left\{r^{i}\left(\left\|x_{k-\rho+t-1}\right\|\right)\right\}
\end{aligned}
$$

with the error $e_{w}^{i}$ defined as

$$
\begin{aligned}
& e_{w}^{i}(k+\tilde{l} \mid k-1)=\left\|w^{i}(k+l \mid k+\tilde{l})-w^{i}(k+l \mid k-1)\right\| \\
& =\left\|w_{k+\tilde{l}}^{i}-w_{k-1}^{i}\right\|
\end{aligned}
$$

For all $l, \tilde{l}=0, \ldots, N^{i}-1$ and with $l \geq \tilde{l}$. The state information $w_{k}^{i}$ is from (4) and is subject to (5).
Proof: Firstly it is shown that (10) holds for $t=0$. The calculation is then repeated for $t=1$, and eventually the general rule for random $t$ will be stated.

For $t=0$ the difference (9) is

$$
\begin{aligned}
\Delta J_{0}^{i} & =J_{N}^{i}(k)-J_{N}^{i *}(k-1) \\
& =\sum_{l=0}^{N^{i}-1}\left\{L^{i}\left(\bar{x}^{i}(k+l \mid k), \bar{u}^{i}(k+l \mid k)\right)\right. \\
& +Q^{i}\left(\bar{x}^{i}(k+l \mid k), w^{i}(k+l \mid k)\right) \\
& -L^{i}\left(\hat{x}^{i}(k+l-1 \mid k-1), u^{i *}(k+l-1 \mid k-1)\right) \\
& \left.-Q^{i}\left(\hat{x}^{i}(k+l-1 \mid k-1), w^{i}(k+l-1 \mid k-1)\right)\right\} \\
& +V^{i}\left(\bar{x}^{i}\left(k+N^{i} \mid k\right)\right)-V^{i}\left(\hat{x}^{i}\left(k+N^{i}-1 \mid k-1\right)\right) \\
& =\sum_{l=0}^{N^{i}-2}\left\{L^{i}\left(\bar{x}^{i}(k+l \mid k), \bar{u}^{i}(k+l \mid k)\right)\right. \\
& -L^{i}\left(\hat{x}^{i}(k+l \mid k-1), u^{i *}(k+l \mid k-1)\right) \\
& +Q^{i}\left(\bar{x}^{i}(k+l \mid k), w^{i}(k+l \mid k)\right) \\
& \left.-Q^{i}\left(\hat{x}^{i}(k+l \mid k-1), w^{i}(k+l \mid k-1)\right)\right\} \\
& +L^{i}\left(\bar{x}^{i}\left(k+N^{i}-1 \mid k\right), h^{i}\left(\bar{x}^{i}\left(k+N^{i}-1 \mid k\right)\right)\right. \\
& -L^{i}\left(x_{k-1}^{i}, u_{k-1}^{i}\right)-Q^{i}\left(x_{k-1}^{i}, w_{k-1}^{i}\right) \\
& +Q^{i}\left(\bar{x}^{i}\left(k+N^{i}-1 \mid k\right), w^{i}\left(k+N^{i}-1 \mid k\right)\right) \\
& +V^{i}\left(\bar{x}^{i}\left(k+N^{i} \mid k\right)\right) \\
& -V^{i}\left(\hat{x}^{i}\left(k+N^{i}-1 \mid k-1\right)\right)
\end{aligned}
$$

Where $\bar{x}^{i}(k+l+1 \mid k+m)$ is the state of the subsystem $i$ at time step $k+l+1$ with $l \in \mathbb{Z}_{\geq 0}$ and $m \in\left[0, N^{i}-1\right]$ while using a feasible control input from (8). It is important to note that since stability of the nominal system is considered, the predicted state $\hat{x}(\cdot)$ and the "feasible" state $\bar{x}(\cdot)$, computed at the same time-step are coinciding.

Using the inequality of Assumption 3, the following result can be obtained

$$
\begin{aligned}
& V^{i}\left(\bar{x}^{i}\left(k+N^{i} \mid k\right)\right)-V^{i}\left(\bar{x}^{i}\left(k+N^{i}-1 \mid k\right)\right) \\
& +L^{i}\left(\bar{x}^{i}\left(k+N^{i}-1 \mid k\right), h^{i}\left(\bar{x}^{i}\left(k+N^{i}-1 \mid k\right)\right)\right) \\
& \left.+Q^{i}\left(\bar{x}^{i}\left(k+N^{i}-1 \mid k\right), w^{i}\left(k+N^{i}-1 \mid k\right)\right)\right) \leq 0
\end{aligned}
$$

Since, nominal stability is considered in this case, we have

$$
V^{i}\left(\bar{x}^{i}\left(k+N^{i}-1 \mid k\right)\right) \equiv V^{i}\left(\hat{x}^{i}\left(k+N^{i}-1 \mid k-1\right)\right)
$$

From (8) we have $\bar{u}^{i}(k+l \mid k)=u^{i *}(k+l \mid k-1)$ for $l=$ $0, \ldots, N^{i}-2$, so imposing this control law, for $m=0$ to system (1), it yields

$$
\begin{aligned}
& L^{i}\left(\bar{x}^{i}(k+l \mid k), \bar{u}^{i}(k+l \mid k)\right) \\
& =L^{i}\left(\hat{x}^{i}(k+l \mid k-1), u^{i *}(k+l \mid k-1)\right) \forall l=0, \ldots, N^{i}-2
\end{aligned}
$$

Notice that using Assumption 2 as well as (11), we obtain

$$
\begin{aligned}
& Q^{i}\left(\bar{x}^{i}(k+l \mid k), w^{i}(k+l \mid k)\right) \\
& -Q^{i}\left(\hat{x}^{i}(k+l \mid k-1), w^{i}(k+l \mid k-1)\right) \leq \\
& \left\|Q^{i}\left(\cdot, w^{i}(k+l \mid k)\right)-Q^{i}\left(\cdot, w^{i}(k+l \mid k-1)\right)\right\| \leq \\
& L_{q w}^{i}\left\|w^{i}(k+l \mid k)-w^{i}(k+l \mid k-1)\right\| \leq L_{q w}^{i} e_{w}^{i}
\end{aligned}
$$


Substituting (11), (13), (14), (15), (16) to (12) and utilizing Assumption 1, the following is derived

$$
\begin{aligned}
\Delta J_{0}^{i} & \leq-L^{i}\left(x_{k-1}^{i}, u_{k-1}^{i}\right)-Q^{i}\left(x_{k-1}^{i}, w_{k-1}^{i}\right) \\
& +\sum_{l=0}^{N^{i}-2}\left\{L_{q w}^{i} e_{w}^{i}(k+l \mid k-1)\right\} \\
& \leq\left(N^{i}-2\right) L_{q w}^{i} e_{w}^{i}(k \mid k-1)-r^{i}\left(\left\|x_{k-1}^{i}\right\|\right)
\end{aligned}
$$

For $t=1$ the difference (9) becomes

$$
\begin{aligned}
\Delta J_{1}^{i} & =\vec{J}_{N}^{i}(k+1)-J_{N}^{i *}(k-1) \\
& =\sum_{l=0}^{N^{i}-1}\left\{L^{i}\left(\bar{x}^{i}(k+l+1 \mid k+1), \bar{u}^{i}(k+l+1 \mid k+1)\right)\right. \\
& -L^{i}\left(\hat{x}^{i}(k+l-1 \mid k-1), u^{i *}(k+l-1 \mid k-1)\right) \\
& +Q^{i}\left(\bar{x}^{i}(k+l+1 \mid k+1), w^{i}(k+l+1 \mid k+1)\right) \\
& \left.-Q^{i}\left(\hat{x}^{i}(k+l-1 \mid k-1), w^{i}(k+l-1 \mid k-1)\right)\right\} \\
& +V^{i}\left(\bar{x}^{i}\left(k+N^{i}+1 \mid k+1\right)\right) \\
& -V^{i}\left(\hat{x}^{i}\left(k+N^{i}-1 \mid k-1\right)\right) \\
& =\sum^{i}-3 \\
& -L^{i}\left(\hat{x}^{i}(k+l+1 \mid k-1), u^{i *}(k+l+1 \mid k-1)\right) \\
& +Q^{i}\left(\bar{x}^{i}(k+l+1 \mid k+1), w^{i}(k+l+1 \mid k+1)\right) \\
& \left.-Q^{i}\left(\hat{x}^{i}(k+l+1 \mid k-1), w^{i}(k+l+1 \mid k-1)\right)\right\} \\
& +L^{i}\left(\bar{x}^{i}\left(k+N^{i}-1 \mid k+1\right), h^{i}\left(\bar{x}^{i}\left(k+N^{i}-1 \mid k+1\right)\right)\right. \\
& +Q^{i}\left(\bar{x}^{i}\left(k+N^{i}-1 \mid k+1\right), w^{i}\left(k+N^{i}-1 \mid k+1\right)\right. \\
& -L^{i}\left(x_{k-1}^{i}, u_{k-1}^{i}\right)-Q^{i}\left(x_{k-1}^{i}, w_{k-1}^{i}\right) \\
& +L^{i}\left(\bar{x}^{i}\left(k+N^{i} \mid k+1\right), h^{i}\left(\bar{x}^{i}\left(k+N^{i} \mid k+1\right)\right)\right. \\
& +Q^{i}\left(\bar{x}^{i}\left(k+N^{i} \mid k+1\right), w^{i}\left(k+N^{i} \mid k+1\right)\right. \\
& -L^{i}\left(x_{k}^{i}, u_{k}^{i}\right)-Q^{i}\left(x_{k}^{i}, u_{k}^{i}\right) \\
& +V^{i}\left(\bar{x}^{i}\left(K+N^{i}-1 \mid k+1\right)\right) \\
& +V^{i}\left(\bar{x}^{i}\left(k+N^{i}+1 \mid k+1\right)\right)-V^{i}\left(\bar{x}^{i}\left(k+N^{i} \mid k+1\right)\right) \\
& -V^{i}\left(\hat{x}^{i}\left(k+N^{i}-1 \mid k-1\right)\right)+V^{i}\left(\bar{x}^{i}\left(k+N^{i} \mid k+1\right)\right) \\
& -V^{i}\left(\bar{x}^{i}\left(K+N^{i}-1 \mid k+1\right)\right) \\
& { }^{i}(10+1)
\end{aligned}
$$

Using similar arguments as in the case of $t=0$, it can be concluded that the difference $\Delta J_{1}^{i}$ is bounded by

$$
\begin{aligned}
\Delta J_{1}^{i} \leq & -L^{i}\left(x_{k-1}^{i}, u_{k-1}^{i}\right)-Q^{i}\left(x_{k-1}^{i}, w_{k-1}^{i}\right) \\
& -L^{i}\left(x_{k}^{i}, u_{k}^{i}\right)-Q^{i}\left(x_{k}^{i}, u_{k}^{i}\right) \\
& +\sum_{l=0}^{N^{i}-3}\left\{L_{q w}^{i} e_{w}^{i}(k+1+l \mid k-1)\right\} \\
& \leq\left(N^{i}-3\right) L_{q w}^{i} e_{w}^{i}(k+1 \mid k-1) \\
& -r^{i}\left(\| x_{k-1}^{i}||\right)-r^{i}\left(\| x_{k}^{i}||\right)
\end{aligned}
$$

From the above it can be concluded using the same procedure, that for random $t \in\left[0, N^{i}-1\right]$ the difference $\Delta J_{t}^{i}=\vec{J}_{N}^{i}(k+t)-J_{N}^{i *}(k-1)$, is given from (10), and hence the proof is completed.

System (1), subject to (2), which satisfies the Assumptions $1,2,3$, is ISS stable with respect to measurement errors of the neighboring agents, under an NMPC strategy. This can be concluded by the optimality of the solution that results to

$$
\begin{aligned}
& J_{N}^{i *}(k)-J_{N}^{i *}(k-1) \leq \Delta J_{0}^{i} \\
& \leq\left(N^{i}-2\right) L_{q w}^{i} e_{w}^{i}(k \mid k-1)-r^{i}\left(\left\|x_{k-1}^{i}\right\|\right)
\end{aligned}
$$

Notice that $J_{N}^{i *}$ is an ISS Lyapunov function of the system (1) under an NMPC framework. This result has been proven in [5] relaying to similar assumptions as in this paper, thus the proof is omitted due to space limitations.

Remark 1: The error (11) can be seen as the error between the predicted and the actual trajectory of the neighboring agents. From equation (11) considering $\tilde{l}=0$, then $w^{i}(k+l \mid k)$ and $w^{i}(k+l \mid k-1)$ are the predicted states of the neighbors at time $k+l$. If we set $w_{k+l}^{i}$ to be the actual state of the systems at time $k+l$, it can be proven that

$e_{w}^{i}(k \mid k-1)=\left\|w^{i}(k+l \mid k)-w_{k+l}^{i}-\left(w^{i}(k+l \mid k-1)-w_{k+l}^{i}\right)\right\|$

which is the difference on the errors between the predicted and the real trajectories of the neighboring agents.

\section{B. Triggering Condition for the NMPC}

In the following, the triggering condition will be provided. Consider that at time $t$ an event is triggered. In order to maintain the ISS property (20) of the system, the Lyapunov function $J_{N}^{i *}(\cdot)$ must be decreasing. Suppose that the error is restricted to satisfy

$$
\left(N^{i}-2\right) L_{q w}^{i} e_{w}^{i}(k \mid k-1) \leq \sigma r^{i}\left(\left\|x_{k-1}^{i}\right\|\right)
$$

with $0<\sigma<1$. Plugging in (21) to (20) we get

$$
J_{N}^{i *}(k)-J_{N}^{i *}(k-1) \leq(\sigma-1) r^{i}\left(\left\|x_{k-1}^{i}\right\|\right)
$$

This suggests that provided $\sigma<1$, the ISS property of the system is still guaranteed.

This triggering rule states that when (21) is violated, the OCP is solved again using the current measurement of the state, as the initial state. If (21) is not violated, the control law from (8) is used for $m=0$.

The triggering rule (21), is only valid in the first step. In order to ensure that the system remains stable while using control law (8) for $m \geq 0$ some additional restrictions for the difference (9) must be stated. According to [12] and the proof of Theorem 1, optimality of the solution is not necessary to guarantee convergence of the closed-loop system. Thus, in order to maintain stability we must ensure that $\Delta J_{t}^{i}$ is strictly decreasing for all $m \geq 0$. Hence, the system can use the control law (8), as long as

$$
\Delta J_{t+1}^{i} \leq \Delta J_{t}^{i}
$$

In this case the convergence of the closed-loop system is guaranteed.

Consequently, the triggering rule can be stated as

$$
\left(N^{i}-t-2\right) L_{q w}^{i} e_{w}^{i}(k+t \mid k-1) \leq \sigma \sum_{\rho=0}^{t}\left\{r^{i}\left(\left\|x_{k-\rho+t-1}^{i}\right\|\right)\right\}
$$


and

$$
\begin{aligned}
& \left(N^{i}-t-2\right) L_{q w}^{i} e_{w}^{i}(k+t \mid k-1)-\sigma r^{i}\left(\left\|x_{k+t}^{i}\right\|\right) \leq \\
& \left(N^{i}-t-1\right) L_{q w}^{i} e_{w}^{i}(k+t-1 \mid k-1)
\end{aligned}
$$

The next OCP is triggered whenever condition (24a) or (24b) is violated. Note that it must hold that $N^{i} \geq 2$. This is a necessity since for prediction horizons $N^{i}<2$, the controller would only provide one step ahead, thus this would result triggering at every time step.

The previous analysis guarantees that the closed loop system will have the same convergence properties as in [5]. However, the OCP in the case of this paper is not calculated at each time instant, but only when the triggering condition is violated. Thus the convergence to a compact set and ultimate boundedness properties of [5] are preserved in the eventtriggered formulation:

Theorem 1: Consider a locally controlled agent $\mathscr{A}^{i}$ for all $i=1, \ldots, M$ with dynamics described by (1), subject to (2), and assume also that the previously presented Assumptions $1,2,3$, hold. Then the NMPC control law given by (6a)(6e) with the neighboring' state information (4) to be subject to (5), along with the triggering rule (24a)-(24b) drives the closed loop system towards a compact set where it is ultimately bounded.

\section{Delays}

When large scale systems are considered, it is expected that there will be a delay during the exchange of the information between the cooperating agents. A triggering condition of each agent of the distributed dynamic system will be defined in the following, in the presence of communication delays.

Assume that agent $\mathscr{A}^{i}$ receives from each neighboring agent $\mathscr{A}^{j} \in \mathscr{G}^{i}$ the value of its state with a delay of $\Delta_{i j}$, which is integer valued. The delayed state information of the neighbors, received by agent $\mathscr{A}^{i}$, is

$$
w_{k-\Delta_{i j}}^{i} \triangleq \operatorname{col}\left(x_{k-\Delta_{i j}}^{j}, j \in G^{i}\right)
$$

Assume that $\Delta_{i j}$ is such that

$$
\left\|w_{k}^{i}-w_{k-\Delta_{i j}}^{i}\right\| \leq \gamma_{i j}
$$

If delays are present, then (10) is modified as follows

$$
\begin{aligned}
\Delta J_{t}^{i} & \leq\left(N^{i}-t-2\right) L_{q w}^{i} e_{w d}^{i}(k+t \mid k-1) \\
& -\sum_{\rho=0}^{t}\left\{r^{i}\left(\left\|x_{k-\rho+t-1}\right\|\right)\right\}
\end{aligned}
$$

with

$$
e_{w d}^{i}(k+t \mid k-1)=\left\|w_{k+t-\Delta_{i j}}^{i}-w_{k-1-\Delta_{i j}}^{i}\right\|
$$

Notice that using the reverse triangle inequality, it yields that

$$
\begin{aligned}
& e_{w}^{i}(k+t \mid k-1)-e_{w d}^{i}(k+t \mid k-1) \\
& =\left\|w_{k+t}^{i}-w_{k-1}^{i}\right\|-\left\|w_{k+t-\Delta_{i j}}^{i}-w_{k-1-\Delta_{i j}}^{i}\right\| \\
& \leq\left\|w_{k+t}^{i}-w_{k-1}^{i}-w_{k-1-\Delta_{i j}}^{i}+w_{k+t-\Delta_{i j}}^{i}\right\| \\
& \leq\left\|w_{k+t}^{i}-w_{k+t-\Delta_{i j}}^{i}\right\|+\left\|w_{k-1}^{i}-w_{k-1-\Delta_{i j}}^{i}\right\| \\
& \leq 2 \gamma_{i j}
\end{aligned}
$$

Thus,

$$
e_{w}^{i}(k+t \mid k-1) \leq e_{w d}^{i}(k+t \mid k-1)+2 \gamma_{i j}
$$

Substituting (28) to (26), it can be obtained that

$$
\begin{aligned}
\Delta J_{t}^{i} & \leq\left(N^{i}-t-2\right) L_{q w}^{i}\left(e_{w d}^{i}(k+t \mid k-1)+2 \gamma_{i j}\right) \\
& -\sum_{\rho=0}^{t}\left\{r^{i}\left(\left\|x_{k-\rho+t}\right\|\right)\right\}
\end{aligned}
$$

Finding the triggering condition in the presence of communication delays using similar approach as in the previous section, is straightforward and is omitted.

\section{EXAMPLE}

In this section, a simulated example of the proposed eventbased framework is presented. Three agents moving in $\mathbb{R}^{2}$ is considered. Each one of the agents is controlled by a local MPC controller while exchanging state information with the neighboring agents without delays.

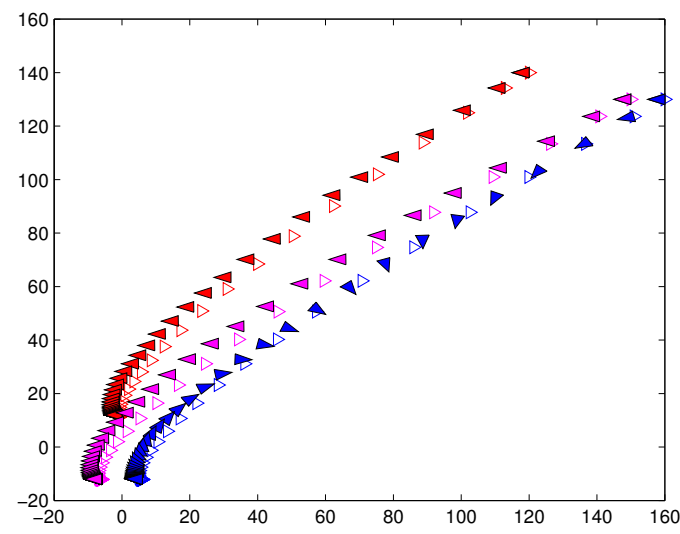

Fig. 1. Trajectories of the team of agents. The filled triangles represent the agents under the event-based NMPC, while the empty triangles represent the agents under time-based NMPC.

The objective of the agents is to reach a desired configuration, while they keep some relative distance between them. For illustrative purposes, the numerical values of the parameters of the system that was taken into account, are taken as in [6], where the classic time-driven MPC was considered, i.e., a controller that runs synchronously in time. The simulation results are reported in Fig. 1 where the trajectories of the three agents are depicted. The filled triangles represent the agents under the event-based framework and the empty triangles represent the agents under classic time-driven MPC. Note, that the orientation of the agents is only depicted in the event-driven case. It can be witnessed that the event-driven as well as the time-driven approach results in comparable performance, i.e., clearly in both cases there is coordinated behavior of the team of the UAV's and collision was always avoided.

The next simulation represents the trajectory of a single agent, in the same cooperative scenario. In Fig.2(a), the trajectory of the agent is shown in both the event-driven 

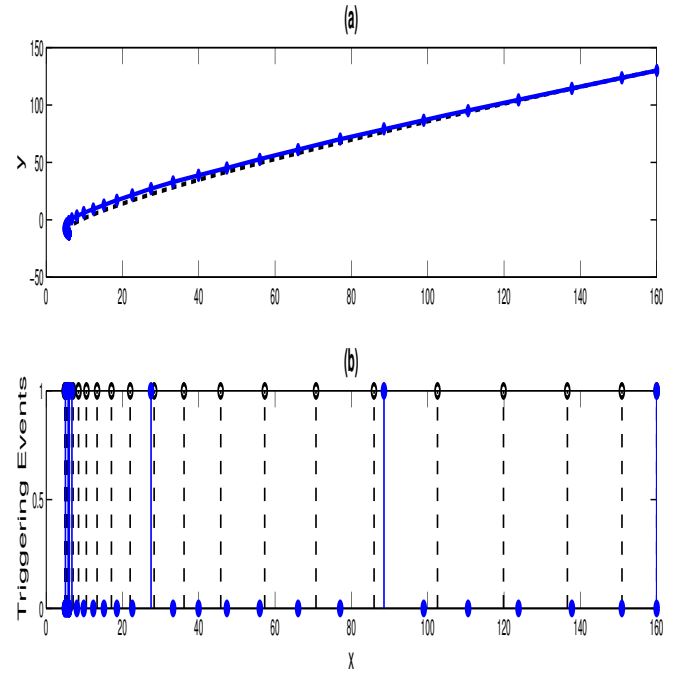

Fig. 2. (a) Trajectory of an agent. The blue solid line represents the trajectory of the agent under the event-based NMPC, while the black dashed line, represents the trajectory of the same agent, under the classic NMPC. (b)Triggering instants. When the triggering axis has the value 1 , the $\mathrm{OCP}$ of the NMPC is triggered. For value 0, the NMPC law is implemented on the system in an open-loop fashion. The blue solid stems the triggering instants of the event-based NMPC, while the black dashed stems represent the sampling instants of the classic time-based NMPC.

and the time-driven case. Figure 2(b) depicts the triggering instants. The black dashed line represents the triggering instants in the time-driven case and the blue solid line depicts the event-triggered policy. In this example, it is evident that the inter-calculation times are strictly larger than one. Namely, with the event-triggered strategy the control updates are significantly lower. Moreover, in both cases, the final configurations of the agents were reached in the same number of sampling instants.

\section{CONCLUSiOnS}

In this paper, an event-based framework for the control of a team of cooperating distributed agents under NMPC controllers was proposed and analyzed. The event-based formulation consists of triggering the solution of the OCP of the NMPC, only when an event occurs. During the interevent period the control sequence provided from the previous triggering event is used in an open-loop fashion. This evenbased scheme is favorable in a number of occasions, because it is possible to reduce the number of times the control law should be computed. This results to the alleviation of the energy consumption.

Future work involves finding a triggering condition in a similar cooperative NMPC framework, however in this case the triggering condition should depend only on local information of the agent and the event-broadcasting state and the predicted state information of the neighboring agents. This event-based approach should be able to reduce the load on the communication medium in addition to agents' energy consumption.

\section{REFERENCES}

[1] K. Åström. Event based control. Analysis and Design of Nonlinear Control Systems, pages 127 - 147, 2008.

[2] D.V. Dimarogonas and E. Frazzoli. Distributed event-triggered control strategies for multi-agent systems. 47th Allerton Conference on Communication, Control, and Computing, 2009.

[3] A. Eqtami, D.V. Dimarogonas, and K.J. Kyriakopoulos. Event- triggered control for discrete-time systems. American Control Conference, pages $4719-4724,2010$.

[4] A. Eqtami, D.V. Dimarogonas, and K.J. Kyriakopoulos. Eventtriggered strategies for decentralized model predictive controllers. IFAC World Congress, 2011.

[5] E. Franco, L. Magni, T. Parisini, M. Polycarpou, and D. Raimondo. Cooperative constraint control of distributed agents with nonlinear dynamics and delayed information excange: A stabilizing recedinghorizon approach. IEEE Trans. Autom. Control, 53(1):324-338, 2008.

[6] E. Franco, Th. Parisini, and M.M. Polycarpou. Cooperative control of discrete-time agents with delayed information exchange: a receding horizon approach. 43th IEEE Conf. Decision and Control, pages 4274 $-4279,2004$

[7] L. Grune, J. Pannek, and K. Worthmann. A prediction based control scheme for networked systems with delays and packet dropouts. 48th IEEE Conf. Decision and Control, pages 537 - 542, 2009.

[8] W.P.M.H. Heemels, J.H. Sandee, and P.P.J. Van Den Bosch. Analysis of event-driven controllers for linear systems. International Journal of Control, 81(4):571-590, 2007.

[9] Y. Iino, T. Hatanaka, and M. Fujita. Event-predictive control for energy saving of wireless networked control system. American Control Conference, pages 2236-2242, 2009.

[10] M. Mazo Jr. and P. Tabuada. Decentralized event-triggered control over wireless sensor/ actuator networks. arXiv:1004.0477v2, 2011

[11] T. Keviczky, F. Borrelli, and G.J. Balas. Decentralized receding horizon control for large scale dynamically decoupled systems. Automatica, 42(13):2105-2115, 2006.

[12] D. Limon Marruedo, T. Alamo, and E.F. Camacho. Input-to-state stable mpc for constrained discrete-time nonlinear systems with bounded additive uncertainties. 41st IEEE Conf. Decision and Control, pages $4619-4624,2002$

[13] D.M. Raimondo, L. Magni, and R. Scattolini. Decentralized mpc of nonlinear systems: An input-to-state stability approach. International Journal of Robust and Nonlinear Control, 17(17):1651 - 1667, 2007.

[14] A. Richards and J. How. Decentralized model predictive control of cooperating uavs. 43th IEEE Conf. Decision and Control, pages 4286 $-4291,2004$

[15] R. Scattolini. Architectures for distributed and hierarchical model predictive control - a review. Journal of Process Control, 19:723731, 2009.

[16] J. Sijs, M. Lazar, and W.P.M.H. Heemels. On integration of eventbased estimation and robust mpc in a feedback loop. Proceedings of the 13th ACM international conference on Hybrid systems: computation and control, pages 31-40, 2010.

[17] P. Tabuada. Event-triggered real-time scheduling of stabilizing control tasks. IEEE Transactions on Automatic Control, 52(9):1680-1685, 2007.

[18] P. Varutti and R. Findeisen. On the synchronization problem for the stabilization of networked control systems over nondeterministic networks. American Control Conference, pages 2216 - 2221, 2009.

[19] X. Wang and M.D. Lemmon. Decentralized event-triggered broadcasts over networked control systems. HSCC '08:Proc. of the 11th International Conference on Hybrid Systems: Computation and Control, pages 674-677, 2008.

[20] X. Wang and M.D. Lemmon. Event-triggering in distributed networked systems with data dropouts and delays. HSCC '09:Proc. of the 12th International Conference on Hybrid Systems: Computation and Control, 52:366-380, 2009.

[21] H. Yu, F. Zhu, and P. J. Antsaklis. Event-triggered cooperative control for multi-agent systems based on passivity analysis. Tech. report, Department of Electrical Engineering, University of Notre Dame, http://www.nd.edu/ isis/techreports/isis-2010-001.pdf, 2010. 